\title{
Clinical outcomes and prognostic factors of transcatheter aortic valve implantation in bicuspid aortic valve patients
}

\author{
Sung-Han Yoon, Rahul Sharma, Tarun Chakravarty, Hiroyuki Kawamori, Yoshio Maeno, Masaki \\ Miyasaka, Takahiro Nomura, Tomoki Ochiai, Sharjeel Israr, Tanya Rami, Mamoo Nakamura, Wen Chen, \\ Raj R. Makkar
}

Department of Interventional Cardiology and Cardiothoracic Surgery, Cedars-Sinai Heart Institute, Los Angeles, CA, USA

Correspondence to: Raj R. Makkar, MD. Department of Interventional Cardiology and Cardiothoracic Surgery, Cedars-Sinai Heart Institute, 8700 Beverly Blvd, Los Angeles, CA 90048, USA. Email: Raj.Makkar@cshs.org.

\begin{abstract}
Background: The purpose of this study was to evaluate the outcomes of transcatheter aortic valve replacement (TAVR) in patients with bicuspid aortic valve stenosis (AS).

Methods: From April 2012 and December 2016, 108 patients with bicuspid AS underwent TAVR using the Sapien XT (34 patients) and Sapien 3 (74 patients) valves. Procedural and clinical outcomes were assessed according to VARC-2 criteria and compared between the two devices.

Results: In the overall cohort, the majority of patients were male (71.3\%) with an intermediate surgical risk and a mean Society of Thoracic Surgeons (STS) score of 5.2\%. Compared to the Sapien XT group, the Sapien 3 group had a significantly lower STS score $(3.3 \% \pm 2.0 \%$ vs. $6.7 \% \pm 3.6 \% ; \mathrm{P}=0.001)$. Compared to the Sapien XT group, the Sapien 3 group had a significantly lower rate of moderate or severe paravalvular leak ( $2.7 \%$ vs. $14.7 \% ; \mathrm{P}=0.03$ ) and higher device success (97.3\% vs. 82.4\%; $\mathrm{P}=0.006)$. There were no significant differences between the two groups in terms of 30-day all-cause mortality, stroke, life-threatening bleeding, major vascular complication and acute kidney injury (stage 2 or 3). Cumulative all-cause mortality at 1-year follow-up was $6.9 \%$. There were no significant differences in cumulative event rates for all-cause mortality at 1 -year follow-up between the two groups ( $9.4 \%$ vs. $4.6 \%$; log-rank $\mathrm{P}=0.47)$. By univariate analysis, major vascular complication was significantly associated with overall all-cause mortality [hazard ratios (HR): 7.57; 95\% confidence interval (CI): 1.51-37.86; $\mathrm{P}=0.014]$.

Conclusions: TAVR using the balloon-expandable valves provided acceptable procedural and clinical outcomes in patients with bicuspid AS. The new-generation Sapien 3 valves showed improved procedural outcomes compared to the early-generation Sapien XT valves.
\end{abstract}

Keywords: Aortic stenosis; transcatheter aortic valve implantation; bicuspid aortic valve

Submitted Jul 24, 2017. Accepted for publication Aug 28, 2017.

doi: 10.21037/acs.2017.09.03

View this article at: http://dx.doi.org/10.21037/acs.2017.09.03

\section{Introduction}

Transcatheter aortic valve replacement (TAVR) has evolved from a novel technology to an established therapy for high-risk patients with severe symptomatic aortic valve stenosis (AS). A number of studies in the literature have demonstrated the safety and efficacy of TAVR, and more than 250,000 patients have been treated with this technology (1-9). Although randomized trials have established TAVR as the standard treatment in inoperable patients and a reasonable option in high surgical risk patients, these trials excluded congenital bicuspid AS due to its unique morphological features such as heavily calcified leaflets, large annulus, calcified raphe, and dilated ascending aorta.

The clinical evidence of TAVR in bicuspid AS is limited to small series (10-13). Based on data from previous registries, the proportion of patients with bicuspid AS may 
reach $2 \%$ to $6 \%(14,15)$. As the indication of TAVR has been expanding to younger and lower surgical-risk patients with increasing frequency of bicuspid AS, there has been increasing concern regarding the clinical outcomes of TAVR in bicuspid AS population (7-9,16-21). In addition, technological advances in the devices used have significantly improved outcomes of TAVR. Therefore, we aim to evaluate the clinical outcomes of TAVR in bicuspid AS, taking into consideration successive device generations.

\section{Methods}

\section{Study design and patient population}

Between April 2012 and December 2016, 120 consecutive patients with bicuspid AS underwent TAVR at the Cedars Sinai Medical Center. In the present study, 108 patients treated with the balloon-expandable transcatheter valves were included, and 12 treated with the other transcatheter valves were excluded. The institutional review board approved this study and all patients provided written informed consent for TAVR and the use of anonymous clinical, procedural, and follow-up data for research. The institutional review board waived the need for written patient informed consent for retrospective analysis of clinically acquired and anonymized data.

\section{Bicuspid aortic valve}

Bicuspid aortic valve morphology was classified as previously described by Sievers et al. according to the number of cusps and the presence of raphes, as well as spatial position and symmetry of raphes and cusps (19). Type 0 was assigned to morphologies characterized by the presence of two symmetric leaflets/cusps and one commissure without evidence of a raphe. Type 1 was assigned to valve morphologies with one raphe, and type 2 when two raphes were present. All participating centers reviewed and subsequently confirmed the diagnosis and classification of bicuspid AS. When both transesophageal echocardiography and pre-procedural computed tomography was performed, patients were excluded if the diagnosis of bicuspid aortic valve was not consistent or remained speculative.

\section{MDCT aortic root dimensions}

Images were post-processed offline at the Cedars Sinai Medical Center core laboratory, using dedicated TAVR planning software (3mensio Structural Heart software, version 8.1, 3mensio Medical Imaging BV, Bilthoven, The Netherlands). The mid-systolic phase of the cardiac cycle was selected and the measurement of the aortic annulus dimensions including diameter (maximum and minimum), area, and perimeter, was performed in the double oblique transverse projection. This projection includes the nadir of all three aortic cusps and perpendicular to the aortic root axis, as previously described $(22,23)$. Ellipticity and area oversizing were calculated as (maximum annulus diameter)/ (minimum annulus diameter) $\times 100$, and [(prosthesis nominal area)/(annulus measured area) -1$] \times 100$, respectively.

\section{Study devices and procedure}

Patients were selected for TAVR after discussions by the multidisciplinary heart team. The multidisciplinary heart team determined the optimal access site. We adopted a transfemoral-first approach policy with criteria for performing a nontransfemoral approach based on the heart team's consideration of the size, calcification, and atheroma of the aortoiliofemoral artery. Device sizes were selected based on three-dimensional, multidetector-row computed tomography-based annular measurements or transesophageal echocardiogram assessment. All TAVR procedures were conducted in accordance with local guidelines using standard techniques via transfemoral, transapical, or transaortic access, and the Sapien XT and Sapien 3 (Edwards Lifesciences, Irvine, California, USA) were implanted (24-29).

\section{Endpoints and definitions}

The primary endpoint of the present study was device success. Secondary endpoints were 30-day major clinical endpoints using the Valve Academic Research Consortium (VARC) 2 criteria (30) as well as all-cause mortality at 1-year follow-up. For the purpose of this study, device success was assessed according to the VARC 2 criteria as followings: absence of (I) procedure-related death (II) moderate or severe paravalvular leak; (III) second valve implantation; (IV) mean gradient more than $20 \mathrm{mmHg}$. Other endpoints included permanent pacemaker insertion, procedureand device-related complications, and echocardiographic assessment of the valve and cardiac function at discharge. The severity of regurgitation was qualitatively assessed and graded using transthoracic echocardiography at each institution according to established guidelines (30). 


\begin{tabular}{|c|c|c|c|c|}
\hline Age, years & $74.4 \pm 10.6$ & $76.7 \pm 10.4$ & $73.3 \pm 10.6$ & 0.11 \\
\hline Male & $77(71.3)$ & $26(76.5)$ & $51(68.9)$ & 0.42 \\
\hline NYHA functional class IV & $39(36.1)$ & $20(58.8)$ & $19(25.7)$ & 0.001 \\
\hline STS score, \% & $5.2 \pm 3.4$ & $6.7 \pm 3.6$ & $3.3 \pm 2.0$ & 0.001 \\
\hline Diabetes mellitus & $28(25.9)$ & $8(23.5)$ & $20(27.0)$ & 0.70 \\
\hline Creatinine, mg/dL & $1.1 \pm 1.0$ & $1.1 \pm 0.3$ & $1.2 \pm 1.2$ & 0.85 \\
\hline Hypertension & $92(85.2)$ & $26(76.5)$ & $66(89.2)$ & 0.08 \\
\hline Chronic lung disease & $32(29.6)$ & $11(32.4)$ & $21(28.4)$ & 0.67 \\
\hline Prior PCl & $17(15.7)$ & $5(14.7)$ & $12(16.2)$ & 0.84 \\
\hline Prior CABG & $14(13.0)$ & 7 (20.6) & $7(9.5)$ & 0.11 \\
\hline \multicolumn{5}{|l|}{ Echocardiographic findings } \\
\hline Mean gradient, $\mathrm{mmHg}$ & $45.3 \pm 14.4$ & $46.4 \pm 16.0$ & $44.8 \pm 13.7$ & 0.58 \\
\hline Aortic valve area, $\mathrm{cm}^{2}$ & $0.6 \pm 0.2$ & $0.6 \pm 0.2$ & $0.6 \pm 0.2$ & 0.11 \\
\hline LVEF, \% & $53.0 \pm 18.0$ & $48.9 \pm 18.4$ & $54.8 \pm 17.6$ & 0.12 \\
\hline
\end{tabular}

EuroSCORE, European System for Cardiac Operative Risk Evaluation; LVEF, left ventricular ejection fraction; NYHA, New York Heart Association; PCI, percutaneous coronary intervention; STS, Society of Thoracic Surgeons.

\section{Statistical analysis}

For the purpose of this study, the outcomes of TAVR with the Sapien XT and Sapien 3 were compared. Continuous variables are presented as mean \pm standard deviation and compared using the Student's $t$-test or Mann-Whitney $\mathrm{U}$ test. Categorical variables are presented as counts or percentages, and compared using the chi-square or Fisher exact test. Statistically significant variables with a $\mathrm{P}$ value of $<0.10$ by univariate analysis were identified as predictors of all-cause mortality. The proportional hazards assumption was confirmed by examination of logarithmic [$\log$ (survival)] curves and by testing of partial (Shoenfeld) residuals, and no relevant violations were found. Cumulative survival rates were analyzed using the KaplanMeier method and compared using log-rank tests. The unadjusted hazard ratios (HR) and 95\% CI were calculated using the Cox model. All statistical analyses were performed using SPSS software (version 21.0, SPSS, Inc., Chicago, IL,
USA). A two-sided P value of $<0.05$ was considered to be of statistical significance.

\section{Results}

\section{Baseline characteristics}

A total of 108 patients with bicuspid AS underwent TAVR using the Sapien XT (34 patients; 31.5\%) and Sapien 3 (74 patients; $68.5 \%$ ). The baseline characteristics of the study population for the Sapien XT and Sapien 3 groups are shown in Table 1. In the overall cohort, the majority of patients were male $(71.3 \%)$ with intermediate surgical risk [Society of Thoracic Surgeons (STS) score: $5.2 \% \pm 3.4 \%$ ]. Compared to the Sapien XT group, the Sapien 3 group had a significantly lower STS score $(6.7 \% \pm 3.6 \%$ vs. $3.3 \% \pm 2.0 \%$; $\mathrm{P}=0.001)$ and logistic European System for Cardiac Operative Risk Evaluation (EuroSCORE) (20.1\% $17.0 \%$ vs. $10.9 \% \pm 8.5 \% ; \mathrm{P}<0.001$ ) as well as less frequent New York 


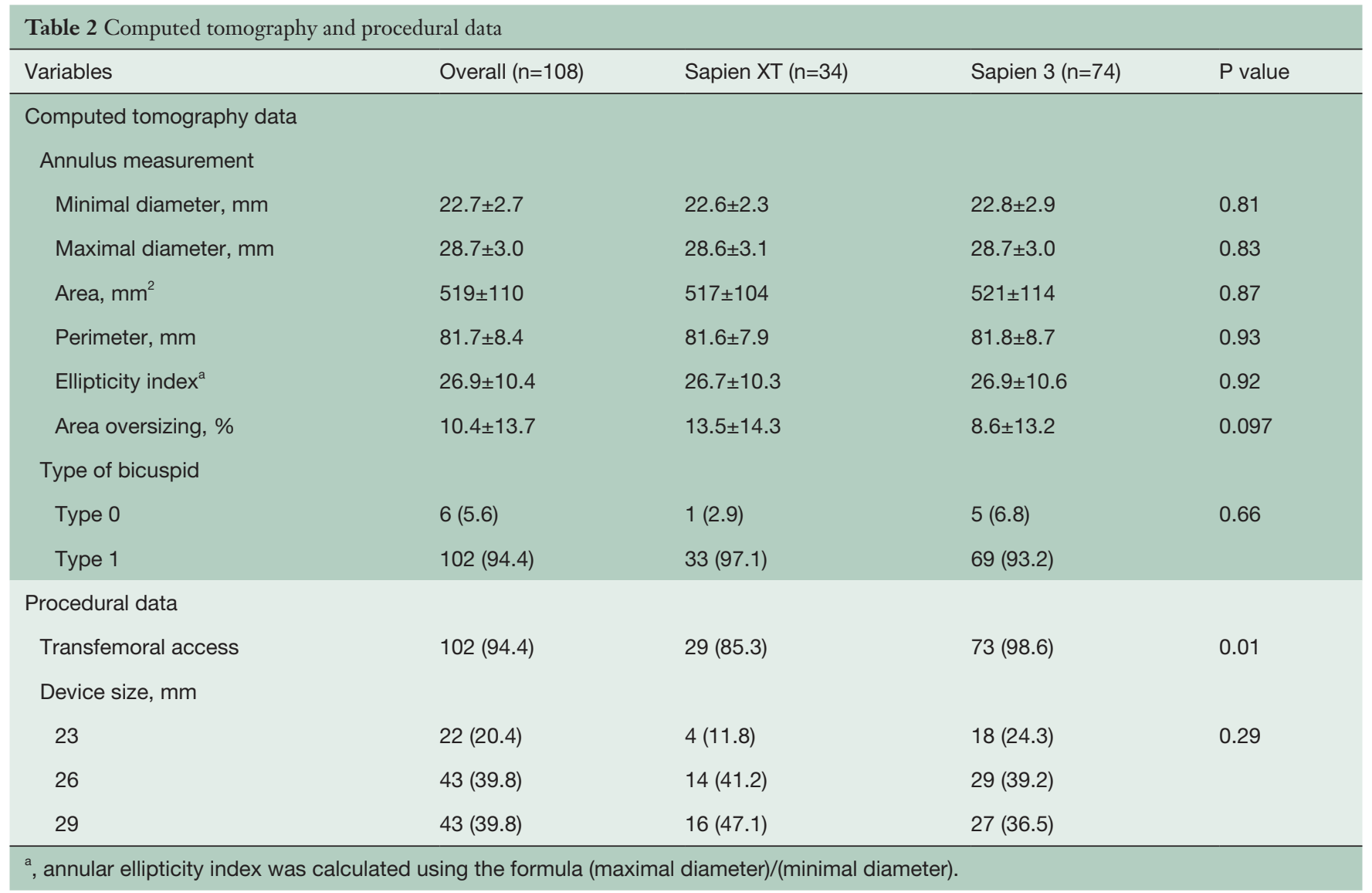

Heart Association functional class IV (58.8\% vs. 25.7\%; $\mathrm{P}=0.001$ ), whereas there were no significant differences between the two groups in other comorbidities.

The computed tomography and procedural findings are presented in Table 2. In terms of annulus dimensions, mean minimal and maximal diameter, area and perimeter were $22.7 \mathrm{~mm}, 28.7 \mathrm{~mm}, 519 \mathrm{~mm}^{2}$, and $81.7 \mathrm{~mm}$, respectively. The type of bicuspid AS was diagnosed in all patients: type 0 in 6 patients (5.6\%) and type 1 in 102 (94.4\%). Transfemoral access was more frequently used in the Sapien 3 group (98.6\% vs. $85.3 \%$; $\mathrm{P}=0.01$ ). Although area oversizing tended to be lower in the Sapien 3 group compared to the Sapien XT group $(8.6 \% \pm 13.2 \%$ vs. $13.5 \% \pm 14.3 \% ; \mathrm{P}=0.097$ ), there was no significant difference in device size between the two groups.

\section{Procedural and clinical outcomes}

The procedural and clinical outcomes of the study population are summarized in Table 3. In the overall cohort, conversion to surgery and annulus rupture occurred in one patient $(0.9 \%)$ and no coronary obstruction was observed. Compared to the Sapien XT group, the Sapien 3 group tended to have a lower rate of second valve implantation $(0.0 \%$ vs. $5.9 \% ; \mathrm{P}=0.097)$ and had a significantly lower rate of paravalvular leak $\geq$ moderate $(2.7 \%$ vs. $14.7 \% ; \mathrm{P}=0.03)$, which resulted in significantly higher device success $(97.3 \%$ vs. $82.4 \% ; \mathrm{P}=0.006$ ).

In terms of 30-day clinical outcomes, there were no significant differences between the two groups in all-cause death $(0.0 \%$ vs. $1.4 \% ; \mathrm{P}>0.99)$, stroke $(2.9 \%$ vs. $5.4 \%$; $\mathrm{P}>0.99)$, life-threatening bleeding $(0.0 \%$ vs. $1.4 \% ; \mathrm{P}>0.99)$, major vascular complication $(2.9 \%$ vs. $6.8 \% ; \mathrm{P}=0.66)$, and stage 2 or 3 acute kidney injury ( $0.0 \%$ vs. $2.7 \%$; $>0.99)$.

\section{Mortality}

Over a mean follow-up period of 360 days (interquartile range, 180-530 days), 10 patients died (seven patients in the Sapien XT group and three patients in the Sapien 3 


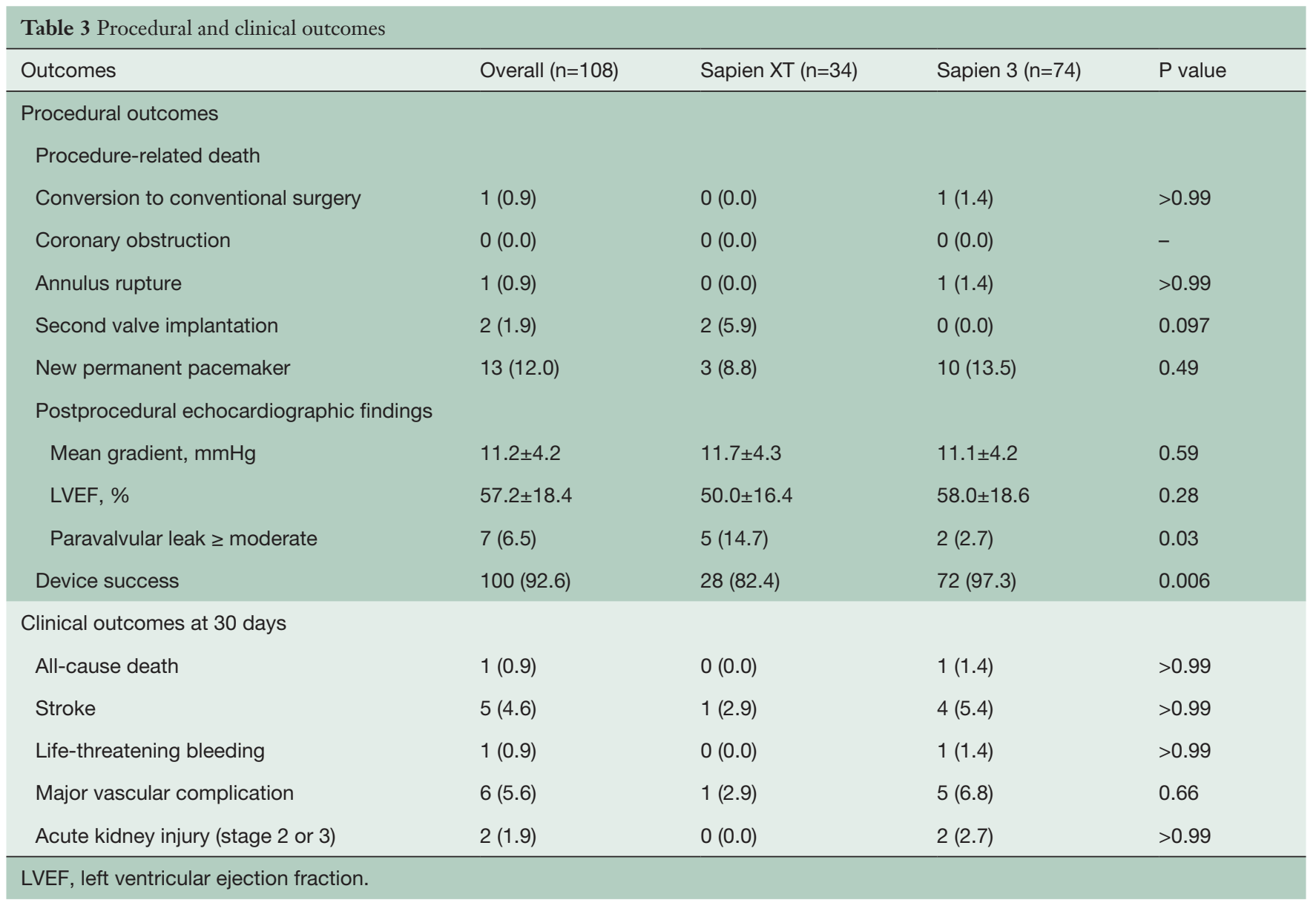

group). In the overall cohort, cumulative all-cause mortality at 1 -year follow-up was $6.9 \%$. There were no differences in all-cause mortality at 1 -year follow-up between the two groups (9.4\% vs. 4.6\%; log-rank $\mathrm{P}=0.47$ ) (Figure 1 ). By univariate analysis, major vascular complication was significantly associated with overall all-cause mortality (HR: 7.57; 95\% CI: $1.51-37.86 ; \mathrm{P}=0.014)$.

\section{Discussion}

The present study showed the safety, efficacy and clinical outcomes of TAVR in patients with bicuspid using the early and new-generation balloon-expandable transcatheter valves. Compared to the early-generation valves, the newgeneration balloon-expandable valves was associated with significantly higher devices success due to lower rates of second valve implantation and paravalvular leak $\geq$ moderate. The overall 1-year all-cause mortality was acceptable without significant difference between the early and new- generation devices.

Recently, two multicenter studies demonstrated the acceptable clinical outcomes of TAVR for bicuspid AS $(10,12)$. Both studies showed the feasibility of TAVR for bicuspid AS with encouraging short- and intermediateterm clinical outcomes. However, high incidences of moderate or severe PVL $(6.0 \%$ and $9.6 \%)$ were reported and showed the limitation of TAVR to treat bicuspid AS with early-generation devices. However, more recent studies showed the improved procedural outcomes with the new-generation devices-moderate or severe paravalvular leak was observed in 0 to $3.8 \%$ with the Sapien $3(11,13,31)$. When using the early-generation balloon-expandable Sapien XT, the oversizing was required to anchor the prosthesis within annulus and approximately more than $10 \%$ area oversizing was needed to avoid significant paravalvular leak. However, as a trade-off, the extreme oversizing leads to an increased risk of annulus rupture, which was as the same with bicuspid AS. The Sapien 3 


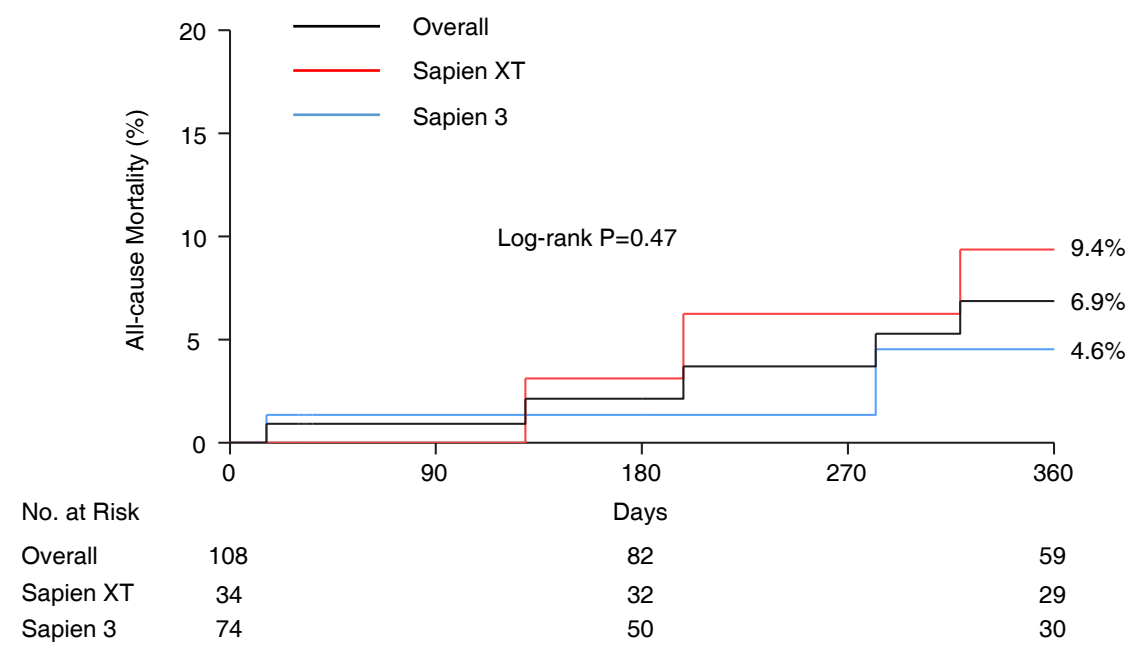

Figure 1 Kaplan-Meier curves for all-cause mortality. The cumulative all-cause mortality rate in patients with bicuspid AS for the overall cohort (black), the Sapien XT (red) and Sapien 3 groups (blue). Event rates were compared by means of the log-rank test. AS, aortic valve stenosis.

with an external sealing cuff allowing for effective sealing and waives the extreme oversizing and mitigates the morphological challenges of bicuspid AS.

In the present study, the STS score and logistic EuroSCORE were lower in Sapien 3 group compared to Sapien XT group. Given all patients were discussed by the multidisciplinary heart team, this may reflect the expanding indication of TAVR to a lower surgical risk population, which has been supported by major randomized trials comparing TAVR versus surgery in intermediate-risk population $(7,32)$. In addition, for the indication for TAVR, we should take into account the factors such as frailty or porcelain aorta, which are not integrated into the surgicalrisk calculation system. In terms of procedural outcomes, the Sapien XT group had a higher rate of moderate or severe paravalvular leak in bicuspid AS (14.7\%), which was numerically higher compared to the reported rates of paravalvular leak after the Sapien XT implantation in tricuspid AS (33). Figure 2 shows the computed tomography, procedural and echocardiographic images of the patient with bicuspid AS treated with the Sapien XT. The $26 \mathrm{~mm}$ Sapien XT provided $15 \%$ area oversizing, but significant calcified annulus and calcified raphe lead to moderate paravalvular leak.

When using the new-generation balloon-expandable Sapien 3, no patients had the second valve implantation, and only 2 patients $(2.7 \%)$ had moderate or severe paravalvular leak. Both cases were type 1 bicuspid AS and performed in early periods of Sapien 3. The cause of paravalvular leak was insufficient oversizing (area oversizing index of 105\%) and extensively calcified leaflet, respectively. Figure 3 showed the computed tomography, procedural and echocardiographic images of the patient with bicuspid AS treated with the Sapien 3. After implantation of the $26 \mathrm{~mm}$ Sapien 3 (17\% area oversizing), only trace paravalvular leak was observed with postprocedural echocardiogram. In our center, the device sizing is based on annulus measurement regardless of the Sapien XT or Sapien 3, as in the case of degenerated tricuspid AS. However, in the case with severely calcified leaflet and/or calcified raphe, device size is cautiously selected. We should take into account all the anatomic factors, including dimensions of annulus, left ventricular outflow tract, sinus of Valsalva, and sino-tubular junction, as well as degree and location of calcification. Similar to tricuspid AS with severely calcified leaflet and/or calcified left ventricular outflow tract, intentional selection of smaller size of bioprostheses should be considered. Importantly, annulus rupture occurred in one patient (1.4\%), which suggests that further study is awaited to establish the guideline regarding device sizing in bicuspid AS. Due to the lack of evidence, the indication of TAVR for bicuspid AS should be considered based on not only the surgical risk but also unfavorable anatomic features.

The present study showed comparable 30-day clinical outcomes between the early and new-generation balloonexpandable valves, probably due to the fewer comorbidities 

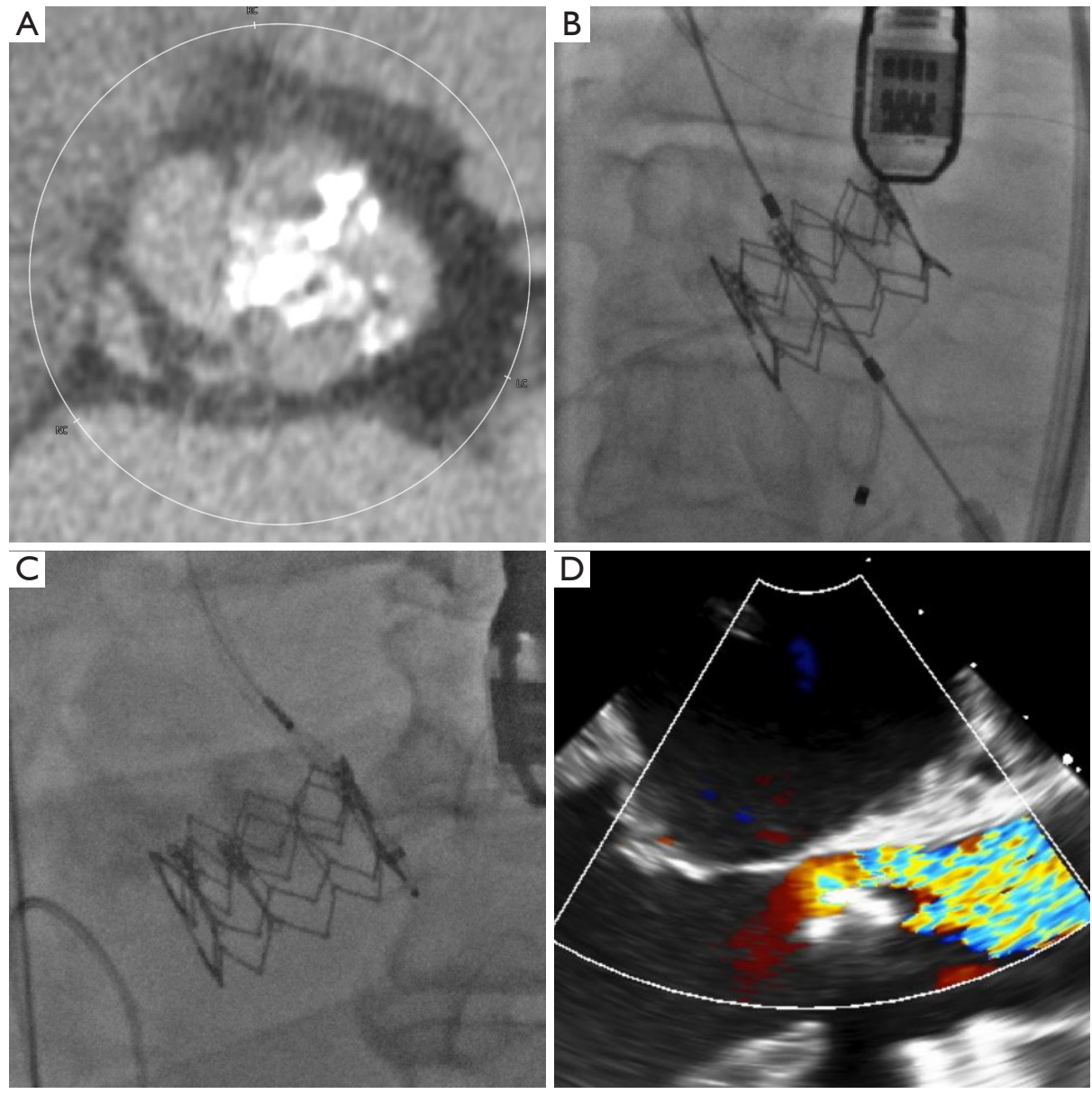

Figure 2 TAVR in bicuspid aortic stenosis with the Sapien XT. The baseline computed tomography showed type 1 bicuspid aortic valve with calcified aortic valve leaflet and raphe (panel A). After the first Sapien XT implantation, severe paravalvular leak was observed by intraprocedural transesophageal echocardiography (panel B). Without improvement of paravalvular leak after balloon dilatation, Amplatzer Vascular Plug II (8 mm) was deployed (panel C). However, postprocedural transesophageal echocardiography showed moderate postprocedural paravalvular leak (panel D). TAVR, transcatheter aortic valve replacement.

in the bicuspid AS population. Despite procedural challenges of TAVR with the early-generation balloonexpandable valves in bicuspid AS, the present study showed similar overall mortality rates between the early and newgeneration valves. This suggests that long-term mortality of patients with bicuspid AS is determined by multiple factors including baseline comorbidities, procedural complications and other major 30-day adverse events.

The present study demonstrated that major vascular complication was associated with increased mortality in bicuspid AS population as well. Although device advancement provided dramatically decreased paravalvular leak less than $5 \%$, the major vascular complication remains still relatively high. Both paravalvular leak and major vascular complication were shown to increase the longterm mortality, and therefore, further effort to decrease the vascular complication is awaited. Furthermore, challenging anatomies of bicuspid AS may lead under-expansion of the prosthesis, which may raise the concern about long-term durability. Given longer life expectancy in those patient populations mandates future studies to evaluate the valve durability for long-term follow-up.

\section{Study limitations}

Limitations of this study include those inherent to a retrospective observational study. Limited sample size leads to difficulty in drawing definitive conclusions, particularly 

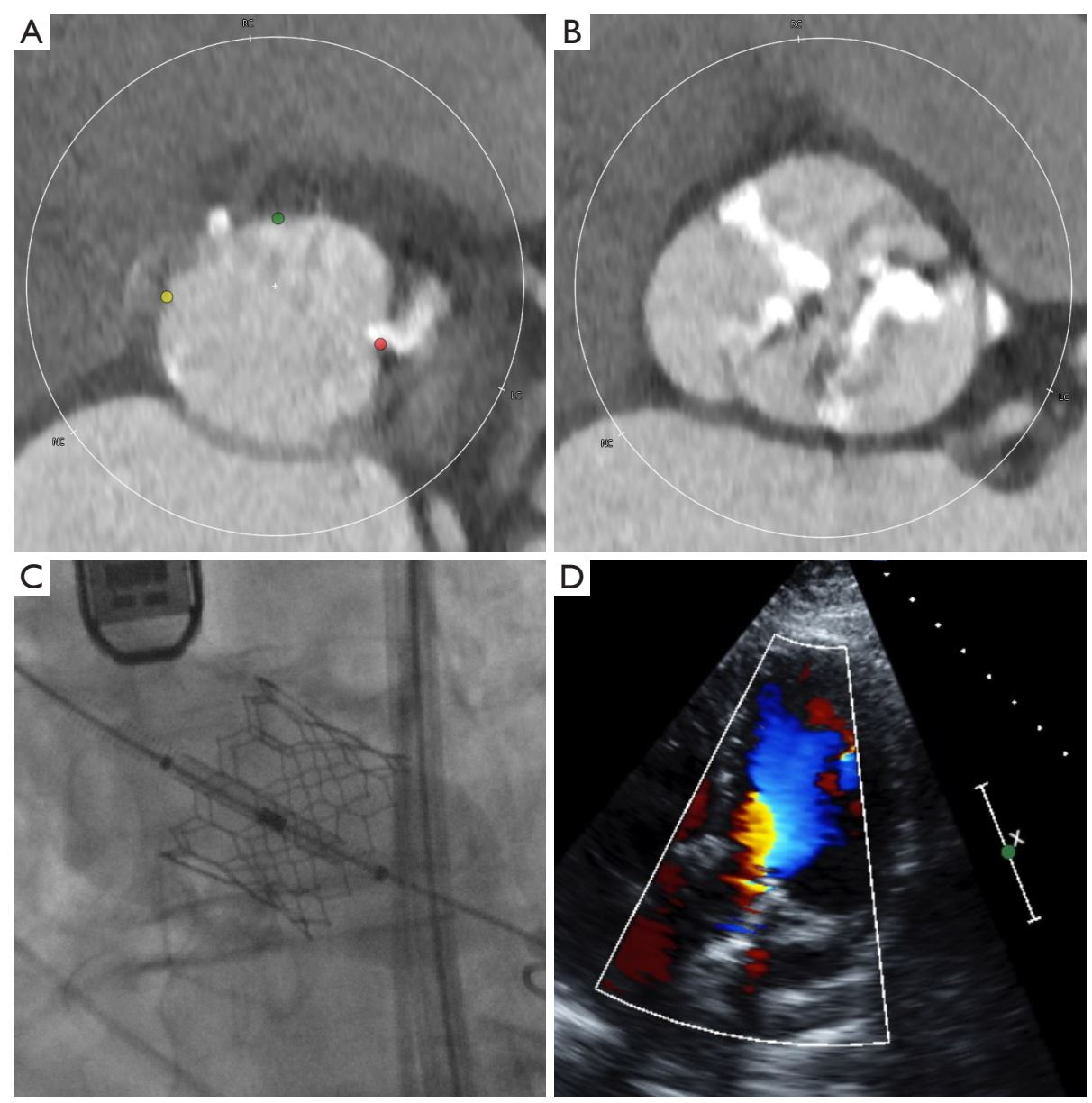

Figure 3 TAVR in bicuspid aortic stenosis with the Sapien 3. The baseline computed tomography showed type 1 bicuspid aortic valve with calcified aortic valve leaflet and raphe (panels A and B). After the successful Sapien 3 implantation, only trace paravalvular leak was observed by postprocedural transthoracic echocardiography (panels $\mathrm{C}$ and D). TAVR, transcatheter aortic valve replacement.

with regard to long-term outcomes. In addition, an inherent learning curve may be associated with the procedural and clinical outcomes.

\section{Conclusions}

TAVR using the balloon-expandable valves provided acceptable procedural and clinical outcomes in patients with bicuspid AS. The new-generation Sapien 3 valves showed improved procedural outcomes compared to the earlygeneration Sapien XT valves.

\section{Acknowledgements}

None.

\section{Footnote}

Conflicts of Interest: Dr. Sharma has served as a proctor for Edwards Lifesciences. Dr. Makkar has received grants from Edwards Lifesciences and personal fees from St. Jude Medical and Medtronic. The other authors have no conflicts of interest to declare.

\section{References}

1. Kodali SK, Williams MR, Smith CR, et al. Two-year outcomes after transcatheter or surgical aortic-valve replacement. N Engl J Med 2012;366:1686-95.

2. Makkar RR, Fontana GP, Jilaihawi H, et al. Transcatheter aortic-valve replacement for inoperable severe aortic stenosis. N Engl J Med 2012;366:1696-704. 
3. Gilard M, Eltchaninoff H, Iung B, et al. Registry of transcatheter aortic-valve implantation in high-risk patients. N Engl J Med 2012;366:1705-15.

4. Hamm CW, Mollmann H, Holzhey D, et al. The German Aortic Valve Registry (GARY): in-hospital outcome. Eur Heart J 2014;35:1588-98.

5. Moat NE, Ludman P, de Belder MA, et al. Long-term outcomes after transcatheter aortic valve implantation in high-risk patients with severe aortic stenosis: the U.K. TAVI (United Kingdom Transcatheter Aortic Valve Implantation) Registry. J Am Coll Cardiol 2011;58:2130-8.

6. Adams DH, Popma JJ, Reardon MJ, et al. Transcatheter aortic-valve replacement with a self-expanding prosthesis. N Engl J Med 2014;370:1790-8.

7. Leon MB, Smith CR, Mack MJ, et al. Transcatheter or Surgical Aortic-Valve Replacement in Intermediate-Risk Patients. N Engl J Med 2016;374:1609-20.

8. Thourani VH, Kodali S, Makkar RR, et al. Transcatheter aortic valve replacement versus surgical valve replacement in intermediate-risk patients: a propensity score analysis. Lancet 2016;387:2218-25.

9. Reardon MJ, Kleiman NS, Adams DH, et al. Outcomes in the Randomized CoreValve US Pivotal High Risk Trial in Patients With a Society of Thoracic Surgeons Risk Score of 7\% or Less. JAMA Cardiol 2016;1:945-9.

10. Mylotte D, Lefevre T, Sondergaard L, et al. Transcatheter aortic valve replacement in bicuspid aortic valve disease. J Am Coll Cardiol 2014;64:2330-9.

11. Perlman GY, Blanke P, Dvir D, et al. Bicuspid Aortic Valve Stenosis: Favorable Early Outcomes With a NextGeneration Transcatheter Heart Valve in a Multicenter Study. JACC Cardiovasc Interv 2016;9:817-24.

12. Yousef A, Simard T, Webb J, et al. Transcatheter aortic valve implantation in patients with bicuspid aortic valve: A patient level multi-center analysis. Int J Cardiol 2015;189:282-8.

13. Yoon SH, Lefevre T, Ahn JM, et al. Transcatheter Aortic Valve Replacement With Early- and New-Generation Devices in Bicuspid Aortic Valve Stenosis. J Am Coll Cardiol 2016;68:1195-205.

14. Mack MJ, Brennan JM, Brindis R, et al. Outcomes following transcatheter aortic valve replacement in the United States. JAMA 2013;310:2069-77.

15. Yoon SH, Ahn JM, Hayashida K, et al. Clinical Outcomes Following Transcatheter Aortic Valve Replacement in Asian Population. JACC Cardiovasc Interv 2016;9:926-33.

16. Piazza N, Kalesan B, van Mieghem N, et al. A 3-center comparison of 1-year mortality outcomes between transcatheter aortic valve implantation and surgical aortic valve replacement on the basis of propensity score matching among intermediate-risk surgical patients. JACC Cardiovasc Interv 2013;6:443-51.

17. Wenaweser P, Stortecky S, Schwander S, et al. Clinical outcomes of patients with estimated low or intermediate surgical risk undergoing transcatheter aortic valve implantation. Eur Heart J 2013;34:1894-905.

18. Walther T, Hamm CW, Schuler G, et al. Perioperative Results and Complications in 15,964 Transcatheter Aortic Valve Replacements: Prospective Data From the GARY Registry. J Am Coll Cardiol 2015;65:2173-80.

19. Sievers HH, Schmidtke C. A classification system for the bicuspid aortic valve from 304 surgical specimens. J Thorac Cardiovasc Surg 2007;133:1226-33.

20. Sabet HY, Edwards WD, Tazelaar HD, et al. Congenitally bicuspid aortic valves: a surgical pathology study of 542 cases (1991 through 1996) and a literature review of 2,715 additional cases. Mayo Clin Proc 1999;74:14-26.

21. Tamburino C, Barbanti M, D'Errigo P, et al. 1-Year Outcomes After Transfemoral Transcatheter or Surgical Aortic Valve Replacement: Results From the Italian OBSERVANT Study. J Am Coll Cardiol 2015;66:804-12 .

22. de Vaan J, Verstraeten L, de Jaegere P, et al. The 3 mensio Valves multimodality workstation. EuroIntervention 2012;7:1464-9.

23. Watanabe Y, Morice MC, Bouvier E, et al. Automated 3-dimensional aortic annular assessment by multidetector computed tomography in transcatheter aortic valve implantation. JACC Cardiovasc Interv 2013;6:955-64.

24. Cribier A, Eltchaninoff H, Tron C, et al. Treatment of calcific aortic stenosis with the percutaneous heart valve: mid-term follow-up from the initial feasibility studies: the French experience. J Am Coll Cardiol 2006;47:1214-23.

25. Webb JG, Pasupati S, Humphries K, et al. Percutaneous transarterial aortic valve replacement in selected high-risk patients with aortic stenosis. Circulation 2007;116:755-63.

26. Ye J, Cheung A, Lichtenstein SV, et al. Transapical transcatheter aortic valve implantation: 1-year outcome in 26 patients. J Thorac Cardiovasc Surg 2009;137:167-73.

27. Grube E, Laborde JC, Gerckens U, et al. Percutaneous implantation of the CoreValve self-expanding valve prosthesis in high-risk patients with aortic valve disease: the Siegburg first-in-man study. Circulation 2006;114:1616-24.

28. Etienne PY, Papadatos S, El Khoury E, et al. Transaortic transcatheter aortic valve implantation with the Edwards SAPIEN valve: feasibility, technical considerations, and 
clinical advantages. Ann Thorac Surg 2011;92:746-8.

29. Bruschi G, de Marco F, Botta L, et al. Direct aortic access for transcatheter self-expanding aortic bioprosthetic valves implantation. Ann Thorac Surg 2012;94:497-503.

30. Kappetein AP, Head SJ, Genereux P, et al. Updated standardized endpoint definitions for transcatheter aortic valve implantation: the Valve Academic Research Consortium-2 consensus document. J Am Coll Cardiol 2012;60:1438-54.

31. Yoon SH, Bleiziffer S, De Backer O, et al. Outcomes in Transcatheter Aortic Valve Replacement for Bicuspid

Cite this article as: Yoon SH, Sharma R, Chakravarty T, Kawamori H, Maeno Y, Miyasaka M, Nomura T, Ochiai T, Israr S, Rami T, Nakamura M, Chen W, Makkar RR. Clinical outcomes and prognostic factors of transcatheter aortic valve implantation in bicuspid aortic valve patients. Ann Cardiothorac Surg 2017;6(5):463-472. doi: 10.21037/acs.2017.09.03
Versus Tricuspid Aortic Valve Stenosis. J Am Coll Cardiol 2017;69:2579-89.

32. Reardon MJ, Van Mieghem NM, Popma JJ, et al. Surgical or Transcatheter Aortic-Valve Replacement in Intermediate-Risk Patients. N Engl J Med 2017;376:1321-31.

33. Athappan G, Patvardhan E, Tuzcu EM, et al. Incidence, predictors, and outcomes of aortic regurgitation after transcatheter aortic valve replacement: meta-analysis and systematic review of literature. J Am Coll Cardiol 2013;61:1585-95. 